\title{
Incidence of drug-induced liver injury in medical inpatients
}

\section{Journal Article}

Author(s):

Meier, Yvonne; Cavallaro, Marzia; Roos, Malgorzata; Pauli-Magnus, Christiane; Folkers, Gerd; Meier, Peter J.; Fattinger, Karin

Publication date:

2005

Permanent link:

https://doi.org/10.3929/ethz-b-000033001

Rights / license:

In Copyright - Non-Commercial Use Permitted

Originally published in:

European Journal of Clinical Pharmacology 61(2), https://doi.org/10.1007/s00228-004-0888-z 


\author{
Yvonne Meier · Marzia Cavallaro · Malgorzata Roos \\ Christiane Pauli-Magnus · Gerd Folkers \\ Peter J. Meier · Karin Fattinger
}

\title{
Incidence of drug-i'nduced liver injury in medical inpatients
}

Received: 7 October 2004/ Accepted: 17 December 2004/Published online: 23 February 2005

(C) Springer-Verlag 2005

\begin{abstract}
Objectives: Drug-induced liver injury (DILI) is a common concern. However, data on DILI epidemiology in inpatients are sparse.

Methods: To investigate the incidence of DILI, we screened all patients in the pharmacoepidemiological inpatient database according to the CIOMS (Council for International Organisation of Medical Science) criteria, which consist of the evaluation of some clinical chemistry laboratory liver parameters (CIOMS laboratory criteria) and the exclusion of any disease-related causes for the liver injury. Thus, only cases with probable or certain causality according to the World Health Organization criteria were included.

Results: Among a total of 6383 patients, liver parameters were determined in 4610, and 489 among them fulfilled the CIOMS laboratory criteria. However, 401 patients had to be excluded because of disease-related liver injury and, thus, the study cohort consisted of 4209 patients at risk for DILI. Among a total of 88 DILI cases, 31 had no documented normal baseline liver parameters and, thus, represented prevalent cases. The remaining 57 represented incident DILI cases. Thus, the incidence of DILI was $1.4 \%(95 \%$ CI $1.0,1.7)$. The drug classes most
\end{abstract}

Y. Meier · C. Pauli-Magnus · P. J. Meier · K. Fattinger $(\bowtie)$

Division of Clinical Pharmacology and Toxicology,

Department of Medicine, University Hospital Zurich,

CH-8091 Zürich, Switzerland

E-mail: fattinge@kpt.unizh.ch

Tel.: +41-1-2552067

Fax: + 41-1-2554411

M. Cavallaro $\cdot$ M. Roos

Biostatistics, ISPM, University of Zurich, Switzerland

Y. Meier · M. Cavallaro

Foundation for Drug Safety and Comprehensive Hospital Drug Monitoring, University Hospital Zurich, CH-8091 Zürich,

Switzerland

G. Folkers

Institute of Pharmaceutical Chemistry,

Department of Applied Biosciences,

Swiss Federal Institute of Technology,

Zurich, Switzerland frequently causing DILI were heparins, antibacterials, tuberculostatics and antineoplastic agents. Among those, antineoplastic agents and tuberculostatics showed the highest incidence. Liver injury was not mentioned among the diagnoses or in the physician's discharge letter in about $52-68 \%$ of all cases.

Conclusion: Approximately 1 in 100 patients develops DILI during hospitalisation in a department of medicine. Incidences of DILI were highest for antineoplastic agents and tuberculostatics. DILI is frequently missed and, therefore, DILI detection by diagnoses will result in misleadingly low incidence rates.

\section{Introduction}

Drug-induced liver injury (DILI) represents a frequently observed adverse drug reaction. Almost all drugs can cause liver enzyme elevations and DILI. However, the majority of drugs exhibit low incidences of hepatic adverse reactions. Therefore, DILI is mostly identified only after broad clinical drug application (phase IV). Wellestablished causes of DILI include non-steroidal antiinflammatory drugs (NSAIDs), antibiotics, antiepileptics, statins, tuberculostatics and herbal medicines [1, 2].

The incidence of DILI has so far been investigated mainly in large outpatient cohorts by screening for hospital admissions due to acute liver injury [3]. In a recent, prospective, population-based cohort study in France, the global outpatient DILI incidence amounted to 14 cases per 100,000 inhabitants (i.e. $0.014 \%$ ) [4]. For inpatients, however, data on incidences and causes of DILI are scarce. The largest published study detected 13 DILI cases by evaluating 147 consecutive inpatients with liver enzyme elevations among 1964 admissions [5]. Thus, DILI was observed overall in $0.7 \%$ of inpatients with $0.2 \%$ admitted to hospital because of DILI [5]. Hence, DILI incidence in the latter inpatient study amounted to $0.5 \%$, suggesting that DILI might develop more frequently in inpatients than in those in ambula- 
tory care. In this study, we used a pharmacoepidemiological database on medical inpatients [6] to determine DILI prevalence at admission and overall, as well as drug-specific DILI incidences in medical inpatients. Furthermore, we also evaluated the type, the outcome and the fraction diagnosed correctly by the physician in charge of the observed DILI cases.

\section{Patients and methods}

\section{Patients}

The SAS/CHDM (Stiftung für Arzneimittelsicherheit/ Comprehensive Hospital Drug Monitoring) project maintains a pharmacoepidemiological database for the purpose of studying adverse drug events in a cohort of medical inpatients [6]. The cohort includes all patients admitted to two to three representative wards at the Departments of Medicine of the University Hospital Zürich and the Kantonsspital (State Hospital) St. Gallen. While the former represents mainly a tertiary referral centre and serves as primary hospital only for some quarters of the city; the latter is a primary city hospital and a secondary referral centre for the northeastern region of Switzerland. In the University Hospital Zurich, the monitored wards belong to the Department of Internal Medicine, where admissions are placed based on available beds independent of the suspected diagnoses, whereas in the State Hospital of St. Gallen the monitored units belong to one of three Divisions of Department of Internal Medicine, preferentially focussing on infectious, endocrine and pulmonary diseases. The exact data recording procedure within the SAS/ CHDM project has been described previously [6]. The database contains structured information on patient characteristics, patient history, clinical events, laboratory results, diagnoses according to International Classification of Disease 10 (ICD10) and detailed drug utilisation prior and during cohort stay. All patients recorded between 1/1996 and 12/2000 were included for analysis.

\section{Case identification}

Patients with DILI were identified according to the CIOMS (Council for International Organizations of Medical Science) criteria, which are based on selected laboratory liver parameters (CIOMS laboratory criteria) and the exclusion of any disease-related causes of liver injury [7]. The CIOMS laboratory criteria require at least two determinations of alanine aminotransferase (ALT) plasma concentrations above $2 \mathrm{~N}(\mathrm{~N}$ stands for upper limit of normal range) (criterion I), conjugated bilirubin above $2 \mathrm{~N}$ (criterion II) or combined increases of aspartate amino-transferase (AST), alkaline phosphatase (AP) and total bilirubin (tBili) with one value above $2 \mathrm{~N}$ (criterion III). All patients meeting the
CIOMS laboratory criteria were assessed by at least two of the authors, one of them an experienced clinical pharmacologist. The liver injury was classified as disease related if (a) a primary liver disease was diagnosed, (b) a recent history of alcohol or cocaine abuse was reported or (c) the time course of the liver parameter elevation was suggestive of an association with an underlying disease such as infectious disease, congestive heart failure, cardiac arrest, acute transitory severe hypotension or autoimmune disease. The assessments were based on all information available in the patients' charts; however, no additional tests could be obtained only for the purpose of this study. So, for example, an ultrasound examination of the liver was available in $51 \%$ (no disease-related causes detected), serologies for hepatitis B/ $\mathrm{C}$ in $16 \%$ (all negative), autoantibodies in $7 \%$ (all negative) and serum ferritin 6\% (all negative) of DILI cases. Since all cases with possible disease-related liver injury were excluded, the final analysis included only DILI cases with probable or certain overall causality according to the World Health Organization (WHO) definitions for adverse drug reactions (see http:// www.who-umc.org/defs.html\#caar).

\section{Type of DILI}

The type of liver injury was determined based on the first laboratory determination corresponding to liver injury according to the CIOMS criteria [7]. Cases with isolated ALT elevation above $2 \mathrm{~N}$ or ALT/AP ratio above 5 (with ALT and AP expressed as multiples of the upper normal limit) were classified as hepatocellular, cases with ALT/AP ratio below 2 as cholestatic and cases with an ALT/AP ratio between 2 and 5 as mixed liver injuries.

\section{Causality assessment for individual drugs}

All medication records were examined, and the time course of the liver parameters was compared with types and dosage histories for all drugs administered before or at the time of liver injury. The type of liver injury and the clinical presentation of the identified cases were compared with those described for the suspected drugs in the literature [8-10]. The causality between drugs and liver injury was determined according to the WHO definitions for adverse drug reactions (see http:// www.who-umc.org/defs.html\#caar): If only one drug could be identified as causative for the DILI, the causality for this drug was classified as probable. If a rechallenge was positive, the causality for the corresponding drug was labelled certain. If more than one drug could have caused the DILI, the causality for all suspected drugs was labelled as possible. Drugs were sorted and grouped according to the ATC classification (http://www.whocc.no/atcddd/, last update 2004). Furthermore, cases with a documented decline of all liver parameters below $2 \mathrm{~N}$ (see definitions above) were 
classified as recovery, whereas cases with documented decline but with liver parameters remaining above $2 \mathrm{~N}$ were labelled as improvement. The label transient was used for cases in which the parameters recovered or improved despite continuation of the causative drug. Cases were labelled as censored if the patient left the cohort while with stable elevated or increasing liver parameters.

Validation of the physicians' assessments

Finally, we checked for each case whether the treating physicians mentioned a diagnosis of or any term related to DILI somewhere in the discharge letters. For cases where DILI was mentioned, we determined in addition which drug(s) was(were) implicated in these documents and made a comparison with the cause(s) identified by our analysis.

Epidemiological and statistical evaluation

All cases with DILI at cohort entry or with no documented normal baseline values prior to DILI development were considered as prevalent cases. In contrast, incident cases required at least one normal liver parameter value prior to DILI development during cohort stay. For statistical analysis, the denominator included all patients with liver parameter determinations during cohort stay and without disease-related liver injury. Exact $95 \%$ confidence intervals $(95 \%$ CI) for incidence and prevalence rates were calculated using the binomial distribution [11]. For patient characteristics and exposure times, median and the first $\left(\mathrm{Q}_{1}\right)$ and third quartiles $\left(\mathrm{Q}_{3}\right)$ are reported.

\section{Results}

\section{Study cohort and identification of DILI cases}

Between January 1996 and December 2000, data from 6383 individual patients were recorded in the SAS/ CHDM database, where $42 \%$ of patients were female and $58 \%$ male. The median $\left(\mathrm{Q}_{1}, \mathrm{Q}_{3}\right)$ age was $61(46,74)$ years. Discharge diagnoses most frequently concerned the cardiovascular system $(65 \%)$, the respiratory system $(30 \%)$, neoplasias $(23 \%)$ and/or infectious diseases $(21 \%)$. The most frequently used drugs were acetaminophen, furosemide, dalteparin and acetylsalicylic acid. The most frequently used antibiotics were amoxicillin/ clavulanic acid, co-trimoxazole and ciprofloxacin. The median $\left(\mathrm{Q}_{1}, \mathrm{Q}_{3}\right)$ duration of cohort stay was $8(5,15)$ days.

Liver parameters, i.e. liver enzymes and/or bilirubin in plasma, were determined in $4610(72 \%)$ of the 6383 patients (Table 1). Among them, 489 patients (i.e. $11 \%$ of those with liver parameter determinations) fulfilled the CIOMS laboratory criteria and 401 patients (i.e. $82 \%$ of patients fulfilling the CIOMS laboratory criteria) suffered from disease-related liver injury caused by cirrhosis (64), hepatic tumours (33), acute hepatotropic viral hepatitis (29), chronic hepatitis B or C (12), alcohol or cocaine abuse (45), decompensated congestive heart failure or transitory severe hypotension (70 patients), acute infections (64), extrahepatic cholestasis (57), autoimmune disease (3) or other causes (24). The latter patients were excluded from the analysis, since the underlying primary liver disease or the disease-related secondary liver damage precluded the adequate detection of DILI. Thus, the study cohort of patients at risk for DILI comprises the 4209 patients with liver parameter determination in whom DILI detection was not precluded by a DILI.

Among the 4209 patients at risk, $88(2.1 \%)$ patients had DILI (Table 1). The diagnosis of DILI was based on an ALT increase above $2 \mathrm{~N}$ (criterion I) in 72 patients, a combined increase of AST, AP and total bilirubin with one value above $2 \mathrm{~N}$ (criterion III) in three patients and both criteria I and III in 13 patients. Criterion II did not contribute to any case identification. Among the 88 DILI patients, 31 were classified as prevalent cases and 57 as incident cases (Table 1).

Table 1 Drug-induced liver injury (DILI) in medical inpatients

Identification of patients with DILI in the SAS/CHDM database

\begin{tabular}{|c|c|c|}
\hline $\begin{array}{l}\text { Total number of } \\
\text { patients screened }\end{array}$ & \multicolumn{2}{|l|}{6383} \\
\hline $\begin{array}{l}\text { Patients with liver } \\
\text { parameter determinations }\end{array}$ & \multicolumn{2}{|l|}{4610} \\
\hline $\begin{array}{l}\text { Patients not fulfilling } \\
\text { CIOMS laboratory criteria }{ }^{\mathrm{a}}\end{array}$ & \multicolumn{2}{|l|}{4121} \\
\hline $\begin{array}{l}\text { Patients fulfilling } \\
\text { CIOMS laboratory criteria }\end{array}$ & \multicolumn{2}{|l|}{489} \\
\hline $\begin{array}{l}\text { Patients with disease-related } \\
\text { liver injury }\end{array}$ & \multicolumn{2}{|l|}{401} \\
\hline Patients with DILI & \multicolumn{2}{|l|}{88} \\
\hline \multicolumn{3}{|c|}{$\begin{array}{l}\text { DILI prevalence at admission and DILI incidence } \\
\text { during hospitalisation }\end{array}$} \\
\hline Patients at risk for DILI ${ }^{\mathrm{b}}$ & 4209 & $100 \%$ \\
\hline Patients with DILI & 88 & $2.1(1.6,2.6) \%$ \\
\hline${\text { Prevalent } \text { cases }^{\mathrm{c}}}$ & 31 & $0.7(0.4,1.0) \%$ \\
\hline Incident cases ${ }^{\mathrm{d}}$ & 57 & $1.4(1.0,1.8) \%$ \\
\hline Hepatocellular & 39 & $0.9(0.6,1.3) \%$ \\
\hline Cholestatic & 14 & $0.3(0.1,0.6) \%$ \\
\hline Mixed & 4 & $0.1(0.0,0.2) \%$ \\
\hline
\end{tabular}

${ }^{a}$ CIOMS laboratory criteria for liver injury: ALT $>2 \times$ upper limit of normal range $(\mathrm{N})$ (criterion I), conjugated bilirubin $>2 \mathrm{~N}$ (criterion II) or aspartate aminotransferase, alkaline phosphatase and total bilirubin $>\mathrm{N}$ provided one of them $>2 \mathrm{~N}$ (criterion III)

$\mathrm{b}$ The study cohort of patients at risk for DILI comprises only patients with liver parameter determinations in whom DILI detection was not precluded by primary or secondary disease related liver injury

c Liver injury already present at admission or no documented normal baseline values during cohort stay prior to the development of DILI

${ }^{\mathrm{d}}$ Liver injury developed during hospitalisation, i.e. documented normal baseline values observed during cohort stay prior to the development of DILI 
Potential risk factors predisposing to DILI

In order to identify potential risk factors predisposing to DILI, patient characteristics of the prevalent and incident DILI cases were compared with the study cohort (Table 2). The percentage of female patients was comparable among cases and the study cohort. Also, the body mass index for the prevalent and the incident DILI cases was comparable with or slightly lower than the study cohort. Except for slightly younger prevalent cases, there were also no significant age or comorbidityindex differences observed. Furthermore, the median number of different drugs per day was comparable between prevalent (6) and incident (6) DILI cases and the study cohort (5). Thus, neither sex, age, polymorbidity nor polypharmacy could be identified as risk factors predisposing to DILI.

Prevalence and types of DILI at admission

Among the 31 prevalent DILI cases (Table 1), 26 (84\%) patients fulfilled the CIOMS criteria already at admission, thus representing reliable prevalent cases. Five $(16 \%)$ additional patients had no documented normal liver parameters during cohort stay prior to DILI development and, therefore, were also classified as prevalent cases. However, this classification remains somewhat tentative, since the latter five patients might possibly correspond to incident cases. Nevertheless, based on the overall cohort of 4209 medical inpatients with liver parameter determinations and the 31 prevalent DILI cases, the overall DILI prevalence at admission amounted to approximately $0.7 \%(0.4,1.0)$ (Table 1$)$.

The overall causality of DILI was probable for all 31 cases. In 29 cases, a single drug was identified as DILI cause and, thus, the causality for the respective drug was also classified as probable. In the other two cases, two drugs could have been causative for DILI and therefore the causality for the respective drugs were classified as possible and the DILI counted for each of the two drugs.

Table 2 Comparison of potential risk factors between drug-induced liver injury (DILI) cases and the study cohort

\begin{tabular}{|c|c|c|c|}
\hline \multirow[t]{2}{*}{$n$} & \multicolumn{2}{|l|}{ DILI cases } & \multirow[t]{2}{*}{ Study cohort } \\
\hline & Prevalent & Incident & \\
\hline & $31(100 \%)$ & $57(100 \%)$ & $4209(100 \%)$ \\
\hline Female & $14(45 \%)$ & $21(37 \%)$ & $1773(42 \%)$ \\
\hline $\operatorname{Age}^{a}$ & $44(35,62)$ & $56(36,70)$ & $61(46,74)$ \\
\hline $\begin{array}{l}\text { Body mass } \\
\text { index }\left(\mathrm{kg} / \mathrm{m}^{2}\right)^{a}\end{array}$ & $24.5(22.0,27.6)$ & $23.8(20.8,27.6)$ & $24.5(21.4,27.7)$ \\
\hline $\begin{array}{l}\text { Comorbidity } \\
\text { index }^{a}\end{array}$ & $1(0,3.5)$ & $2(0,3)$ & $1(0,3)$ \\
\hline $\begin{array}{l}\text { Number of } \\
\text { different } \\
\text { drugs per day }{ }^{a}\end{array}$ & $6(3,8)$ & $6(4,8)$ & $5(3,7)$ \\
\hline
\end{tabular}

${ }^{a}$ a given as median $\left(\mathrm{Q}_{1}, \mathrm{Q}_{3}\right)$
Only four patients were admitted to the hospital because of DILI. The causative drugs were amoxicillin/clavulanic acid, isoniazid, sulfadiazine and Chinese herbs. In the remaining 27 prevalent cases, hospital admission was unrelated to DILI.

The distribution of the prevalent cases to drug classes contributing two or more cases and to types of DILI is given in Fig. 1. Hepatocellular DILI was most frequently observed with heparins (7 cases), NSAIDS (5), anticancer agents (4), statins (2) and tuberculostastics (2). The latter also contributed to cholestatic DILI, together with antibacterials, antimycotics and anticancer drugs. The five NSAID-induced DILI cases were caused by diclofenac (2), ibuprofen, flurbiprofen and nimesulide. DILI was caused just once by ciclosporin, furosemide, gestrinone and Chinese herbs.

\section{Incidence and types of DILI during cohort stay}

Based on the 57 incident cases and the 4209 inpatients at risk, the overall DILI incidence amounted to $1.4 \%$, with a $95 \%$ CI of $(1.0,1.8)$ (Table 1). If instead all patients including those without liver parameter determination were included in the denominator $(n=6383)$, a slightly lower incidence estimate of $1.0 \%(0.7,1.2)$ would have been obtained. Hence, the real DILI incidence in our patient cohort must probably lie somewhere between $0.7 \%$ and $1.8 \%$. According to the CIOMS criteria, liver injury was hepatocellular in $39(0.9 \%)$, cholestatic in 14 $(0.3 \%)$ and mixed in $4(0.1 \%)$ patients.

The overall causality of DILI was certain in 2 and probable in 55 incident cases. In 44 cases with probable causality, a single drug could be identified as the cause of DILI and thus the causality for the respective drug was also classified as probable. In the remaining 11 probable

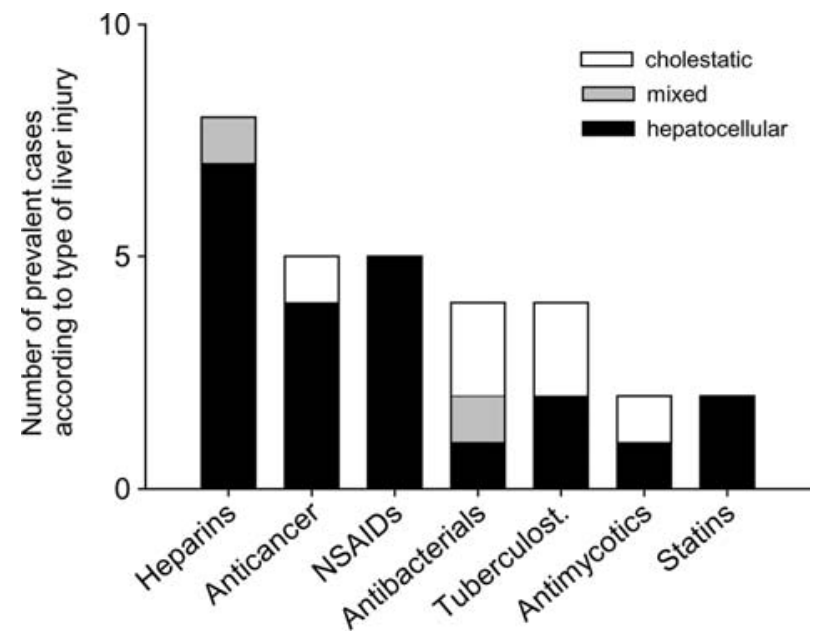

Fig. 1 Causative drugs in 31 prevalent cases of drug-induced liver injury (DILI) according to drug class and type of liver injury. The drugs most commonly causing DILI were heparins (ATC classification: $\mathrm{B} 01 \mathrm{AB}$ ) followed by anticancer agents (L01), non-steroidal anti-inflammatory drugs (NSAIDs, N02B), antibacterials for systemic use (J01), tuberculostatics (J04), antimycotics for systemic use (J02) and statins (C10AA) 
cases, 2 drugs could have been causative for DILI and, therefore, the causality for the respective drugs were classified as possible and the DILI counted for each of the two drugs.

The distribution of the incident cases to drug classes contributing two or more cases and to types of DILI is given in Fig. 2a. The most frequent DILI causes were heparins, antibacterials and anticancer agents. Other DILI causes were antimycotics, tuberculostatics and antivirals. Pravastatin, ciclosporin and mesalazine caused DILI in single cases only. Based on the number of patients exposed, incidence rates were calculated and are given in Fig. 2b. DILI incidence was highest for anticancer agents $(3.8 \%)$ followed by tuberculostatics $(2.4 \%)$. Incidence estimates for heparins, antibacterials, antimycotics and antivirals were comparable and less than $2 \%$.
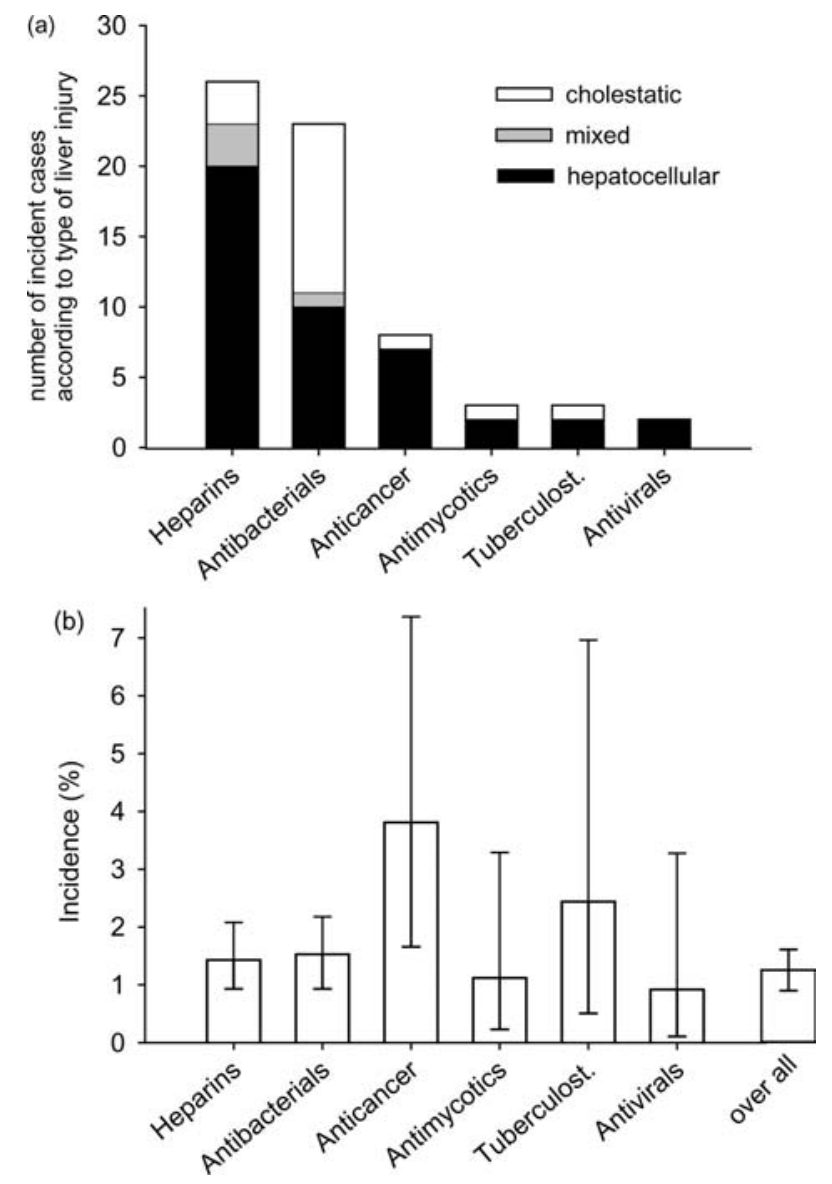

Fig. 2 a Causative drugs in 57 incident cases of drug-induced liver injury (DILI) according to drug class and types of liver injury. The drugs most commonly causing DILI were heparins (ATC classification: $\mathrm{B} 01 \mathrm{AB})$, antibacterials for systemic use (J01), anticancer agents (L01), antimycotics for systemic use (J02), tuberculostatics (J04) and antivirals for systemic use (J05). b Incidence of druginduced liver for selected drug classes. Incidence rates were highest for patients receiving anticancer agents (L01) and amounted to $3.8 \%(95 \%$ CI: 1.6, 7.4), followed by tuberculostatics with an incidence of $2.4 \%(0.5,7.0)$, antibacterials for systemic use with $1.5 \%(0.9,2.2)$, heparins with $1.4 \%(0.9,2.1)$, antimycotics with $1.1 \%(0.2,3.3)$ and antivirals with $0.9 \%(0.1,3.3)$. Values are given as mean $\pm 95 \% \mathrm{CI}$
Incidence of DILI for heparins

In the study cohort, 675 patients were exposed to unfractionated heparin, 1576 to low-molecular-weight heparins (mainly dalteparin) and 427 patients received both types of heparin sequentially. Since 6 incident DILI cases were attributed to unfractionated heparin and 20 to low-molecular-weight heparins, incidence estimates were $0.9(0.3,1.9) \%$ for unfractionated heparin and 1.3 $(0.7,2.0) \%$ for low-molecular-weight heparins. The type of liver injury was mostly hepatocellular (20 of 26, Fig. 2a). Of the 19 patients with documented follow-up, DILI was transient in 11 cases and recovered or improved in 8 cases only after heparin was stopped. Thus, heparin-associated DILI was mostly hepatocellular and transient, and incidences were comparable for both types of heparin.

\section{Antibacterial-associated DILI}

There were 19 incident DILI cases induced by antibacterials. Thorough care was taken in each case to exclude increases of liver parameters caused by the treated infection: in 3 cases, antibacterials were administered prophylactically. In the 16 other cases, liver injury occurred only after clinical and laboratory parameters suggested recovery from infection. The type of liver injury was hepatocellular in 9, mixed in 1 and cholestatic in 9 cases (Fig. 2a). Eleven patients showed recovery, 5 patients exhibited improvement and 11 patients recovered or improved only after antibacterials were stopped. DILI was transient in 4 cases. Beta-lactams were involved in 15 cases, sulphonamides in 2 and lincomycins in 2. The beta-lactams causing DILI were amoxicillin/ clavulanic acid (9 cases), cephalosporins (4), floxacillin (1) and imipenem (1). Based on 571 exposed patients, DILI incidence in amoxicillin/clavulanic acid-treated inpatients amounted to $1.6(0.7,3.0) \%$. Amoxicillin/ clavulanic acid-associated liver injury was cholestatic in 6 patients and hepatocellular in 3 (Table 3). However, in all cases where $\gamma$-glutamyl-transpeptidase was determined, it was elevated severalfold. The amoxicillin/clavulanic acid-associated DILI cases exhibited a lower median age of $44(33,69)$ years compared with a value of $69(52,81)$ years obtained for all amoxicillin/clavulanic acid-exposed patients. Moreover, $42 \%$ of the amoxicillin/clavulanic acid-associated DILI cases and $36 \%$ of the exposed patients were female.

\section{Anticancer agent-associated DILI}

The causative agents in the seven incident anticancer agent-associated DILI cases are given in Table 4. Except for one cholestatic DILI caused by tretinoin, all other cases showed hepatocellular DILI. Three patients showed recovery and two patients exhibited improvement. Antimetabolites were the cause of DILI in 4 cases, 
Table 3 Incident cases with amoxicillin/clavulanic acid-induced liver injury

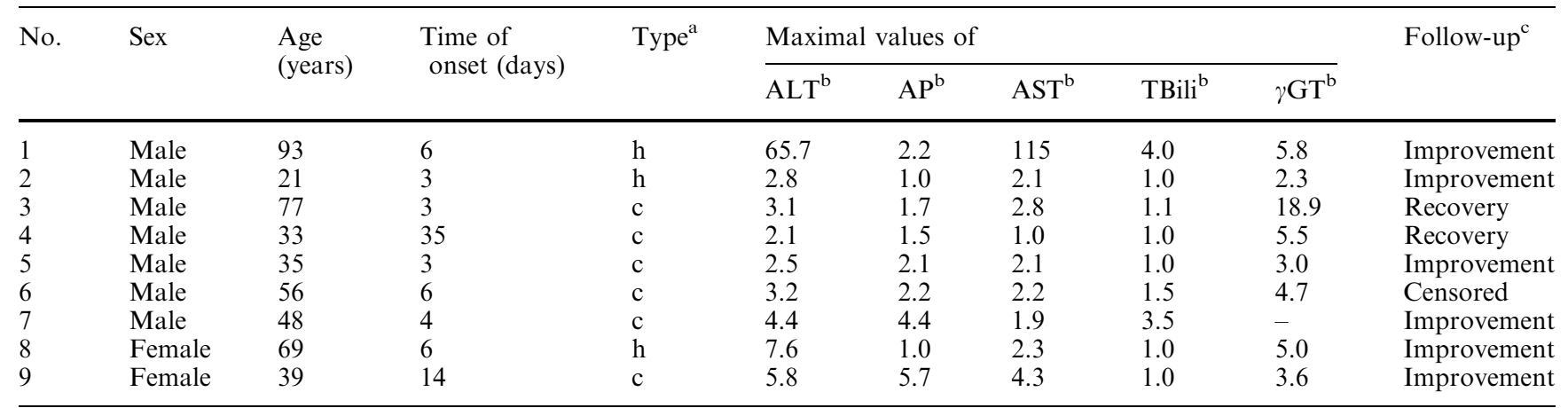

${ }^{a}$ Type of liver injury: $h$ corresponds to hepatocellular, $c$ to cholestatic DILI

${ }^{b}$ Maximal elevations for alanine aminotransferase $(A L T)$, alkaline phosphatase $(A P)$, aspartate amino-transferase $(A S T)$, total bilirubin $(t B i l i)$ and $\gamma$-glutamyl transpeptidase $(\gamma \mathrm{GT})$ in multiples of the upper normal limits. Values below the upper limit are given as 1.0

\begin{abstract}
${ }^{\mathrm{c}}$ The term recovery implies that liver parameters did no longer fulfil CIOMS criteria, whereas the term improvement was used for cases where CIOMS criteria were still fulfilled, but liver parameters started to decrease when patients left the cohort. The term censored stays for patients leaving the cohort with liver parameters still fulfilling CIOMS criteria and no improvement was documented
\end{abstract}

Table 4 Incident cases with liver injury induced by anticancer agents

\begin{tabular}{|c|c|c|c|c|c|c|c|c|c|c|c|}
\hline \multirow[t]{2}{*}{ No. } & \multirow[t]{2}{*}{ Sex } & \multirow[t]{2}{*}{ Age (years) } & \multirow[t]{2}{*}{ Drug } & \multirow[t]{2}{*}{ Time of onset (days) } & \multirow[t]{2}{*}{ Type $^{\mathrm{a}}$} & \multicolumn{5}{|c|}{ Maximal values of } & \multirow[t]{2}{*}{ Follow-up } \\
\hline & & & & & & $\mathrm{ALT}^{\mathrm{b}}$ & $\mathrm{AP}^{\mathrm{b}}$ & $\mathrm{AST}^{\mathrm{b}}$ & $\mathrm{tBili}^{\mathrm{b}}$ & $\gamma \mathrm{GT}^{\mathrm{b}}$ & \\
\hline \multicolumn{12}{|c|}{ Antimetabolites } \\
\hline 1 & Male & 26 & Cytarabine $^{\mathrm{d}}$ & 2 & $\mathrm{~h}$ & 4.4 & 1.0 & 2.2 & 1.3 & 1.5 & $\operatorname{tran} / \mathrm{impr}$ \\
\hline 2 & Male & 15 & Mercaptopurine & 7 & $\mathrm{~h}$ & 2.3 & 1.0 & 1.0 & - & 1.0 & censored \\
\hline 3 & Female & 20 & Methotrexate & 6 & $\mathrm{~h}$ & 4.4 & 1.0 & 1.8 & - & - & censored \\
\hline 4 & Female & 38 & Methotrexate & 4 & $\mathrm{~h}$ & 3.6 & 1.0 & 1.0 & - & 1.0 & recovery \\
\hline \multicolumn{12}{|c|}{ Alkaloids } \\
\hline 5 & Male & 26 & Etoposide $^{\mathrm{d}}$ & 2 & $\mathrm{~h}$ & 4.4 & 1.0 & 2.2 & 1.3 & 1.5 & $\operatorname{tran} / \mathrm{impr}$ \\
\hline 6 & Male & 28 & Vincristine & 5 & $\mathrm{~h}$ & 3.8 & 1.0 & 1.0 & - & 4.1 & recovery \\
\hline \multicolumn{12}{|c|}{ Alkylating agents } \\
\hline 7 & Male & 60 & Cyclo-phospamide & 1 & $\mathrm{~h}$ & 2.3 & 1.0 & 7.0 & 1.0 & 1.0 & recovery \\
\hline \multicolumn{12}{|c|}{ Miscellaneous } \\
\hline 8 & Male & 65 & Tretinoin & 33 & $\mathrm{c}$ & 3.1 & 5.5 & 1.6 & 2.7 & 10.4 & recovery \\
\hline
\end{tabular}

a Type of liver injury: $h$ corresponds to hepatocellular, $c$ to cholestatic according to the CIOMS criteria

${ }^{\mathrm{b}}$ Maximal elevations for alanine aminotransferase $(A L T)$, alkaline phosphatase $(A P)$, aspartate amino-transferase $(A S T)$, total bilirubin $(t B i l i)$ and $\gamma$-glutamyl transpeptidase $(\gamma G T)$ in multiples of the upper normal limits. Values below the upper limit are given as 1.0

${ }^{c}$ The term recovery implies that liver parameters did no longer fulfil CIOMS criteria, whereas the term improvement was used for cases

alkaloids in 2, alkylating agent in 1 and tretinoin also in 1 case. The causality for the drug was probable in 4 and possible in 3 cases. Among the latter, the concomitant drug was another anticancer agent in 1 case. Thus, DILI was mainly hepatocellular and concerned several anticancer agents.

\section{Exposure times before DILI}

Figure 3 gives the exposure times for all incident cases until DILI occurrence, i.e. the number of days elapsed between the first intake of the causative drug until DILI occurred. Exposure times varied from just a few days to over 2 weeks (10 cases). The hepatocellular type where CIOMS criteria was still fulfilled, but liver parameters starts to decrease when patients left the cohort. The term censored stays for patients leaving the cohort with liver parameters still fulfilling CIOMS criteria and no improvement was documented. The term tran/ impr was used for cases in which the parameters improved despite of continuation of the offended drug

d Numbers 1 and 5 correspond to the same patient. Based on the presentation and the clinical course, both, cytarabine or etoposide might have caused the liver injury

outweighs the cholestatic type for short exposure times, whereas for longer exposure times the partition into hepatocellular and cholestatic type is more balanced. Correspondingly, the median $\left(\mathrm{Q}_{1}, \mathrm{Q}_{3}\right)$ exposure times amounted to $4(3,6)$ days for hepatocellular and to $6(4$, 13) for cholestatic DILI cases.

Detection and evaluation of DILI by treating physicians

In order to evaluate DILI detection by the physicians in charge, the diagnoses and the text of the discharge letters of all cases were examined (Table 5). DILI was diag- 


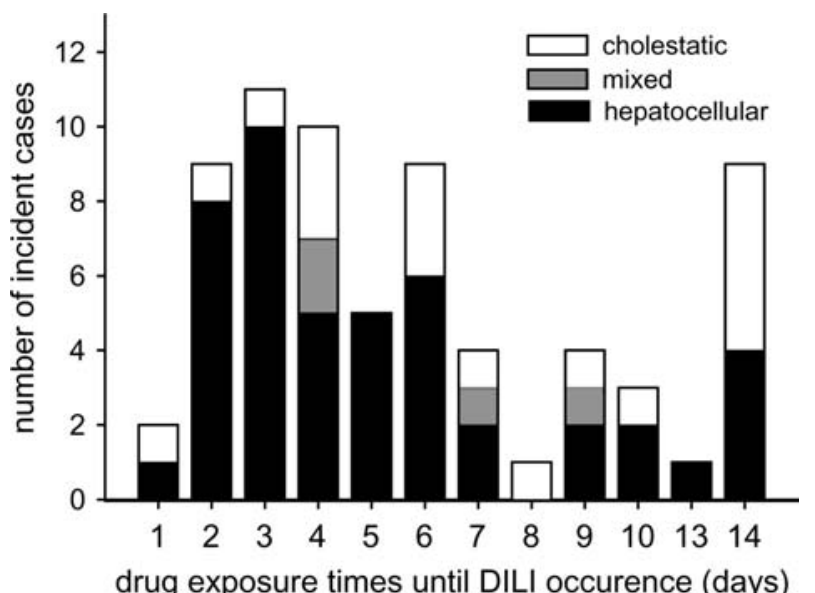

Fig. 3 Time of onset (days) of drug-induced liver injury (DILI) in 57 incident DILI cases. The time of onset was determined as the number of days elapsed between the date at which the suspected drug was started and the occurrence of liver injury

Table 5 Detection and evaluation of drug-induced liver injury (DILI) by the treating physicians

\begin{tabular}{lll}
\hline$n$ & Incident cases & Prevalent cases \\
\hline $\begin{array}{l}\text { DILI diagnosed } \\
\begin{array}{l}\text { DILI mentioned in } \\
\text { discharge letter }\end{array}\end{array}$ & $57(100 \%)$ & $31(100 \%)$ \\
$\begin{array}{l}\text { Causative drug correctly } \\
\text { identified }\end{array}$ & $5(9 \%)$ & $14(45 \%)$ \\
$\begin{array}{l}\text { Wrong drug implicated } \\
\begin{array}{l}\text { Causative drug not } \\
\text { specified }\end{array}\end{array}$ & $9(16 \%)$ & $1(3.0 \%)$ \\
& $3(5.0 \%)$ & $9(29 \%)$ \\
\hline
\end{tabular}

nosed in only $23 \%$ of all incident cases and in $45 \%$ of the prevalent cases. In addition, DILI was mentioned somewhere else in the text in $9 \%$ and $3 \%$, respectively. Thus, a case detection procedure based on the recorded diagnoses or the discharge letters would have missed 60$70 \%$ of all cases. Even if we disregard the 34 missed, mostly transient heparin-associated DILI cases, underreporting still amounts to $40-60 \%$. Interestingly, the physicians in charge noted none of the 5 NSAID-associated DILI cases. Thus, DILI is frequently missed or not reported during routine care in medicine.

\section{Discussion}

In this study of medical inpatients, the DILI prevalence at admission amounted to $0.7 \%$ and the overall incidence during hospitalisation to $1.4 \%$. Neither sex, age, polymorbidity nor polypharmacy predisposed to DILI. Heparins, antibacterials and anticancer drugs were most frequently causative for DILI, whereas incidences were highest for anticancer drugs and tuberculostatics. Of patients identified by screening electronically for the CIOMS laboratory criteria, $82 \%$ exhibited a disease-related liver injury and $18 \%$ a DILI. Furthermore, the physicians in charge missed the majority of DILI diagnoses for incident and prevalent cases.

DILI incidence in medical inpatients was estimated to a value between $0.7 \%$ and $1.8 \%$ with a point estimate of $1.4 \%$. The lower limit originates from the lower 95\% CI if the entire cohort was included, whereas the upper limit corresponds to the upper $95 \% \mathrm{CI}$ if the cohort was limited to patients with liver parameter determinations. Furthermore, $0.7 \%$ of patients were admitted with DILI; thus, overall, $2.1 \%$ exhibited a DILI. This is below the value of $3.7 \%$ [12] in a department of neurology and is three times higher than the value of $0.7 \% \mathrm{ob}-$ tained by evaluating all liver parameter elevations among 1964 consecutive hospitalisations [5]. Thus, DILI occurs commonly in inpatients, especially in neurology and medicine. The marked discrepancy to the low annual incidences in outpatients of $0.014 \%$ [4] might arise due to different case detection methods, i.e. screening laboratory results versus screening diagnoses or evaluating physicians' notifications, and/or be due to differences in age, morbidity and drug exposures.

Among 489 patients, only 88 patients had DILI resulting in a positive predictive value of $18 \%$ for a case detection based on the CIOMS laboratory criteria. This is in line with previous smaller studies in medicine reporting positive predictive values of $12-17 \%$ [13, 14] and slightly lower than the $31-37 \%$ for a department of neurology [12]. These numbers indicate that automatic signal generation would result in too many false-positive alerts, at least if one uses the CIOMS criteria cut-off of $2 \mathrm{~N}$. If the cut-off is increased to $2.5 \mathrm{~N}$ or $3 \mathrm{~N}$, the positive predictive value increases only to $22 \%$ or $20 \%$, respectively. In turn, $28 \%$ or $49 \%$ of DILI cases would be missed. In contrast, if we had relied on DILI detection by the physicians in charge, $52-68 \%$ of cases would have been missed (Table 5). Thus, case detection for a comprehensive DILI epidemiology study can neither be accomplished only by laboratory screening nor by analysing only patients' records, but requires laboratory data for screening combined with a thorough evaluation of positive screenings by a specially trained physician or pharmacist.

One might argue that the high number of DILIs missed by the physicians in charge is not worrisome, since none of the DILI cases developed severe or fatal outcome and most cases did not show clinical symptoms (data not shown). However, one-third of the 72 uncensored patients only recovered after stopping the causing drug, and in $10 \%$ other drugs were identified incorrectly as causative for DILI. These numbers point towards the importance of diagnosing all DILI cases as well as correctly identifying the causative drug(s) to be able to stop the causative drugs and avoid unnecessary withdrawals of essential medications.

Common DILI causes in our study were heparins, antimicrobials, anticancer agents immunosuppressants, statins and NSAIDs. Similar drugs were implicated in a previous study at the Geneva University Hospital in Switzerland [15]. Thus, these drugs seem to represent 
indeed the most common DILI causes at larger hospitals in Switzerland. Without exposure data, heparins and antibacterials would be supposed to be the most frequent DILI causes. However, if we also consider drug exposures, DILI occurs almost twice as frequently with anticancer agents and tuberculostatics as with antibacterials and heparins. This nicely illustrates the importance of exposure data for comparing risk rates among drugs.

Sex, age, polypharmacy and liver disorders are often discussed as predisposing to DILI [14, 16, 17]. Since patients with underlying liver disorders were excluded in this study, we cannot evaluate the influence of liver disease. Interestingly, we did not detect any differences in sex, age, comorbidity and polypharmacy between prevalent and incident DILI cases and the overall study cohort. The observation that even for incident cases polypharmacy was not increased suggests that it is exposure to drugs with high hepatotoxic potential rather than polypharmacy in general that predisposes to DILI. These findings also suggest that predisposition to DILI might rather depend on some pharmacogenetic trait than on concomitant diseases, aging or hormones.

NSAIDs caused none of the incidents, but five of the prevalent cases. An explanation might be that NSAIDs were only sparsely used within the cohort $(17 \%)$, whereas acetaminophen (47\%) and opioids (35\%) were more commonly administered. Interestingly, none of the NSAID-associated prevalent cases was mentioned among the discharge diagnoses or anywhere else in the discharge letters, and all cases improved or recovered only after stopping the NSAID. These findings suggest that outpatient studies using case identification by discharge diagnoses might considerably underestimate DILI incidences.

It is well established that heparins lead frequently to asymptomatic aminotransferase elevations and DILI usually transiently occurring after 5-10 days of treatment [10]. All heparin-associated DILI cases in this study corresponded closely to these characteristics. One randomised controlled trial comparing unfractionated heparin with low-molecular-weight heparin (fraxiparine) reported a significantly higher incidence of transaminase elevations above $3 \mathrm{~N}$ for unfractionated heparin $(3 \%)$ than low-molecular-weight heparin $(0.9 \%)$ [18]. Incidence rates in our study were in the same range, but showed no differences for low-molecular-weight and unfractionated heparin. Thus, we cannot confirm that DILI is less common with low-molecular-weight heparins. Although heparin-induced DILI is usually asymptomatic, a correct diagnosis can be essential for preventing unnecessary withdrawals of essential drugs incorrectly implicated as DILI causes.

Among the antibacterial-associated DILI cases, amoxicillin/clavulanic acid was the most common cause and led to DILI in $1.6 \%$ of exposed patients. In is well known that clavulanic acid can lead to cholestatic liver injury typically after 14 days of treatment $[19,20]$. Also in our study, DILI pattern was cholestatic in two-thirds of the cases and exposures prior DILI varied between
3 days and 35 days. A recent review of all case reports showed that $70 \%$ of the amoxicillin/clavulanic acidassociated DILI cases were male and the average age was 60 years [20]. In contrast, the median age for the amoxicillin/clavulanic acid-associated cases in our study was somewhat lower (44 years) than among amoxicollin/clavulanic acid users (69 years). The fraction of female patients was not different between amoxicillin/ clavulanic acid-associated DILI cases $(36 \%)$ and amoxicillin/clavulanic acid users $(42 \%)$. Thus, our epidemiological analysis does not confirm the predominance of older male patients for amoxicillin/clavulanic acid-associated DILI in case series [20] suggesting that the reported predominance might possibly be caused by higher exposure rates in elderly males. Incidences of amoxicillin/clavulanic acid-associated DILI in our inpatient study were about 100 times higher than the previously reported incidence rates in outpatients of $0.017 \%$, i.e. 1.7 cases per 10,000 prescriptions [19]. Thus, amoxicillin/clavulanic acid-associated DILI is mainly cholestatic and occurs quite commonly in inpatients.

Anticancer agents are usually not mentioned among drugs causing DILI. Nevertheless, in our study, we obtained the highest incidence $(3.8 \%)$ for anticancer agents. Among seven cases, six were hepatocellular suggesting that DILI with anticancer agents usually exhibits a hepatocellular pattern with few exceptions, such as for example tretinoin. The reason why anticancer agent-associated DILI is frequently overlooked might be the severe underlying disease and the high frequency and large variety of expected and tolerated adverse drug effects after chemotherapy.

In conclusion, our study demonstrates that in medical inpatients DILI occurs in approximately one in 100 patients during hospitalisation, which is considerably more common than reported for ambulatory care. Incidences were highest for anticancer agents, tuberculostatics, antibacterials and heparins. Since primary and secondary disease-related liver injuries are also common in this patient collective, screening laboratory results according to the CIOMS laboratory criteria exhibits a low specificity for DILI with a positive predictive value of $18 \%$. However, DILI is frequently missed by the treating physician and therefore its detection by diagnoses will result in misleadingly low incidence rates.

Acknowledgements The project is supported by a grant from the foundation "Stiftung für Arzneimittelsicherheit/Comprehensive Hospital Drug Monitoring". The foundation is supported by Glaxo Smith Kline, Novartis, Roche and the Swiss Medical Association (FMH). Karin Fattinger was supported by the Swiss National Science Foundation (grant 3200-065173.01). There are no conflicts of interests related to this work.

\section{References}

1. Pratt DS, Kaplan MM (2000) Evaluation of abnormal liverenzyme results in asymptomatic patients. $\mathrm{N}$ Engl $\mathrm{J}$ Med $342: 1266-1271$ 
2. Lee WM (1995) Drug-induced hepatotoxicity. N Engl J Med 333:1118-1127

3. Garcia Rodriguez LA, Ruigomez A, Jick H (1997) A review of epidemiologic research on drug-induced acute liver injury using the general practice research data base in the United Kingdom. Pharmacotherapy 17:721-728

4. Sgro C, Clinard F, Ouazir K et al (2002) Incidence of druginduced hepatic injuries: a French population-based study. Hepatology 36:451-455

5. Bagheri H, Michel F, Lapeyre Mestre M et al (2000) Detection and incidence of drug-induced liver injuries in hospital: a prospective analysis from laboratory signals. Br J Clin Pharmacol 50:479-484

6. Fattinger K, Roos M, Vergeres P et al (2000) Epidemiology of drug exposure and adverse drug reactions in two Swiss departments of internal medicine. Br J Clin Pharmacol 49:158-167

7. Benichou C (1990) Criteria of drug-induced liver disorders. Report of an international consensus meeting. J Hepatol $11: 272-276$

8. Morant J, Ruppaner H (2001) Arzneimittelkompendium der Schweiz. Documed, Basel

9. O'Grady JG, Lake JR, Howdle PD (2000) Comprehensive clinical hepatology. Mosby, London

10. Stricker BHC (1992) Drug-induced hepatic injury. Elsevier, Amsterdam

11. Wissenschaftliche Tabellen Geigy. (1980) CIBA-GEIGY AG, Basel

12. Thuermann PA, Windecker R, Steffen J et al (2002) Detection of adverse drug reactions in a neurological department: comparison between intensified surveillance and a computer-assisted approach. Drug Saf 25:713-724
13. Azaz Livshits T, Levy M, Sadan B, Shalit M, Geisslinger G, Brune K (1998) Computerized surveillance of adverse drug reactions in hospital: pilot study. Br J Clin Pharmacol 45:309314

14. Dossing M, Sonne J (1993) Drug-induced hepatic disorders. Incidence, management and avoidance. Drug Saf 9:441-449

15. Oestreicher MK, Desmeules J, Giostra E, Gross B, Hadengue A, Dayer P (1996) Atteintes hepatiques medicamenteuses, il faut y penser! Schweiz Med Wochenschr 126:2094-2096

16. Begaud B (1990) The liver as the target organ for idiosyncratic reactions. In: Jones CANaJK (ed) Idiosyncratic adverse drug reactions. Elsevier Science Publishers, Amsterdam, B.V., pp 85-97

17. Perez Gutthann S, Garcia Rodriguez LA (1993) The increased risk of hospitalizations for acute liver injury in a population with exposure to multiple drugs. Epidemiology 4:496-501

18. Harenberg J, Roebruck P, Heene DL (1996) Subcutaneous lowmolecular-weight heparin versus standard heparin and the prevention of thromboembolism in medical inpatients. The heparin study in internal medicine group. Haemostasis 26:127139

19. Garcia Rodriguez LA, Stricker BH, Zimmerman HJ (1996) Risk of acute liver injury associated with the combination of amoxicillin and clavulanic acid. Arch Intern Med 156:13271332

20. Gresser U (2001) Amoxicillin-clavulanic acid therapy may be associated with severe side effects - review of the literature. Eur J Med Res 6:139-149 